aneurysm - the abnormal segment of subclavian artery should also be excised. Primary anastomosis may be possible when only a short length of vessel has been removed. However, this was not possible in our case and vascular reconstruction with a prosthetic graft was necessary.

This case demonstrates that a cervical rib may cause peripheral and cerebral thromboembolism in the thoracic outlet syndrome. Urgent angiography should be used to identify the site and extent of arterial pathology. Patients need surgical decompression of the thoracic outlet with excision of the segment of abnormal artery.

\section{References}

1. Riddell, D.H. \& Smith, B.M. Thoracic and vascular aspects of thoracic outlet syndrome. Clin Orthop Rel Res 1986, 207: 31-36.

2. Martin, J., Gaspard, D.J., Johnston, P.W., Kohl, R. \& Dietrick, W. Vascular manifestations of the thoracic outlet syndrome. Arch Surg 1976, 111: 779-782.

3. Haimovici, H. Arterial thromboembolism of the upper extremity associated with the thoracic outlet syndrome. $J$ Cardiovasc Surg 1982, 23: 214-220.

4. Bouhoutsos, J., Morris, T. \& Martin, P. Unilateral Raynaud's phenomenon in the hand and its significance. Surgery 1977, 82: $547-551$.

5. McCleery, R.S., Kesterton, J.E., Kirtley, J.A. \& Love, R.B. Subclavius and anterior scalenus muscle compression as a cause of intermittent obstruction of the subclavian vein. Ann Surg 1951, 133: 588-602.

6. Etheredge, S., Wilbur, B. \& Stoney, R.J. Thoracic outlet syndrome. Am J Surg 1979, 138: 175.

7. Judy, K.L. \& Heymann, R.L. Vascular complications of thoracic outlet syndrome. Am J Surg 1972, 123: 521-531.

8. Shucksmith, H.S. Cerebral and peripheral emboli caused by cervical ribs. $\mathrm{Br}$ Med J 1963, ii: 825-837.

\section{Acknowledgements}

We wish to thank Dr J. Reidy, F.R.C.R., consultant radiologist, and Dr A.M. Hanby, M.R.C.Path., consultant histopathologist, Guy's Hospital for help in compiling this case report.

Mr W.R. O'Flynn died suddenly and unexpectedly whilst this manuscript was in preparation. We (P.B. and J.P.) believe this case to be a celebration of his diagnostic and professional skill.

9. Prior, A.L., Wilson, L.A., Gosling, R.G., Yates, A.K. \& Russell, R.R. Retrograde cerebral embolism. Lancet 1979 , 17: $1044-1047$.

10. Cormier, J.M., Amrane, M., Ward, A. \& Laurian, C. Arterial complications of the thoracic outlet syndrome: fifty-five operative cases. J Vasc Surg 1989, 9: 778-787.

11. Clagett, O.T. Presidential address: research and prosearch. $J$ Thorac Cardiovasc Surg 1962, 44: 153-166.

12. Scher, L.A., Veith, F.J. \& Haimovici, H. Staging of arterial complications of cervical rib: guidelines for surgical management. Surgery 1984, 95: 645-649.

13. Heyden, B. \& Vollmar, J. Thoracic outlet syndrome with vascular complications. J Cardiovasc Surg 1979, 20: $531-536$.

14. Roos, D.B. Transaxillary approach for first rib resection to relieve thoracic outlet syndrome. Ann Surg 1966, 163 354-358.

15. Murphy, T.O., Piper, C.A. \& Kanar, E.A. Subclavicular= approach to first rib resection. Am J Surg 1980, 139: 634-636.

\title{
Idiopathic calcification of the basal ganglia
}

\author{
H.F.K. Chiu, L.C.W. Lam ${ }^{1}$, P.P.S. Shum ${ }^{1}$ and K.W. Li ${ }^{2}$
}

Department of Psychiatry, Chinese University of Hong Kong, Shatin, Hong Kong, ${ }^{1}$ Kwai Chung Hospital, Hong Kong and ${ }^{2}$ Prince of Wales Hospital, Hong Kong

Summary: Idiopathic calcification of the basal ganglia is a rare disorder characterized by neuropsychiatric abnormalities, a movement disturbance of parkinsonian and/or choreoathetoid type and dense calcification of the basal ganglia. We report a 60 year old patient diagnosed as having delusional disorder and tardive dyskinesia who was subsequently found to be suffering from idiopathic calcification of the basal ganglia.

\section{Introduction}

Idiopathic calcification of the basal ganglia (ICBG) is a rare disorder characterized by neuropsychiatric

Correspondence: H.F.K. Chiu, M.R.C.Psych.

Accepted: 1 June 1992 abnormalities, a movement disturbance of parkinsonian and/or choreoathetoid type and dense calcification of the basal ganglia. ${ }^{1}$ Its aetiology is unknown but it is familial in some cases. ${ }^{2,3}$ Other causes associated with calcification of the basal 
ganglia include hypoparathyroidism, pseudohypoparathyroidism, pseudopseudohypoparathyroidism, hyperparathyroidism, tuberous sclerosis, various inflammatory and infectious conditions, such as cytomegalic inclusion disease, toxoplasmosis, encephalitis, toxic and anoxic conditions, such as lead intoxication and carbon monoxide poisoning. ${ }^{1}$ Tardive dyskinesia is an involuntary movement disorder associated with prolonged antipsychotic drug use and its prevalence is around $20 \%$ in psychiatric patients. ${ }^{4}$ We report a patient with ICBG presenting with neuropsychiatric manifestations and movement disorder misdiagnosed as delusional disorder with tardive dyskinesia.

\section{Case report}

A 60 year old married Chinese man, born in Malaysia, had no formal education and came to work as a labourer in Hong Kong around 30 years ago. He lost contact with his family in the subsequent years, hence we had no details of his family history. His psychiatric history began at age 55 when he was hospitalized for a brief psychotic episode. He presented with a 2 week history of delusion of reference and delusion of persecution. As a result of his psychotic symptoms, he hit a stranger and then attempted suicide for fear of being arrested by police. After admission into a mental hospital, a diagnosis of delusional disorder was made and he was put on chlorpromazine $50 \mathrm{mg}$ daily. His symptoms subsided after a few weeks and he was subsequently discharged. He attended outpatient treatment irregularly and his compliance to drug treatment was poor.

A year later he manifested delusion of persecution and was hospitalized again. After admission, he was noticed to be dull and slow in mentation, and harboured a delusion that his neighbour would kill him. He was slightly depressed but had no sleep, appetite or weight disturbance. After receiving thioridazine $50 \mathrm{mg}$ daily for a few weeks, his psychotic symptoms subsided. A year later he manifested bucco-linguo-masticatory syndrome and choreoathetoid movements of his limbs and a diagnosis of tardive dyskinesia was made. Antipsychotic drugs were discontinued but the dyskinesia persisted for 3 years.

He was subsequently referred to us for management because of the authors' special interest in movement disorder. On examination, he was slow and retarded. He had no psychotic features but his cognitive function was impaired with poor attention and concentration. He could recall two out of three items after 5 minutes. His clinical picture was consistent with a mild dementia but he refused any formal psychometric tests. Physical examination revealed moderate buccolingual dyskinesia and choreoathetoid movements of his limbs with bradykinesia and rigidity. A computed tomographic (CT) scan of the brain showed extensive bilateral calcification of the globus pallidus (Figure 1). Clinically he had no evidence of hypoparathyroidism, pseudohypoparathyroidism or pseudopseudohypoparathyroidism. Routine laboratory studies including serum calcium and phosphorus levels, thyroid function tests and caeruloplasmin level were normal. A diagnosis of idiopathic basal ganglia calcification with dementia was made.

\section{Discussion}

Dementia, a schizophrenic-like psychosis ${ }^{1,5}$ and organic mood disorder ${ }^{6,7}$ have been described as neuropsychiatric manifestations in ICBG. Cummings et al. ${ }^{1}$ described two distinct manifestations of ICBG. An early-onset type appeared between the ages of 20 and 40 years in which a schizophrenic-like psychosis was noted at initial presentation. Late-onset ICBG presented between the ages of 40 and 60 years with dementia and a movement disorder of the parkinsonian and/or choreoathetoid type. König ${ }^{8}$ suggested that time of occurrence of the calcification might be important as depositions in early life may produce oligophrenia while at later dates, mood changes occurred and at still later dates, dementia syndromes predominated. In many cases of ICBG, the globus pallidus was a prominent area of involvement but no correlation of symptomatology with localization or extent of calcification was found. ${ }^{8}$

Our patient had bilateral calcification of the globus pallidus, a movement disorder in the form of parkinsonian and choreoathetoid type and a

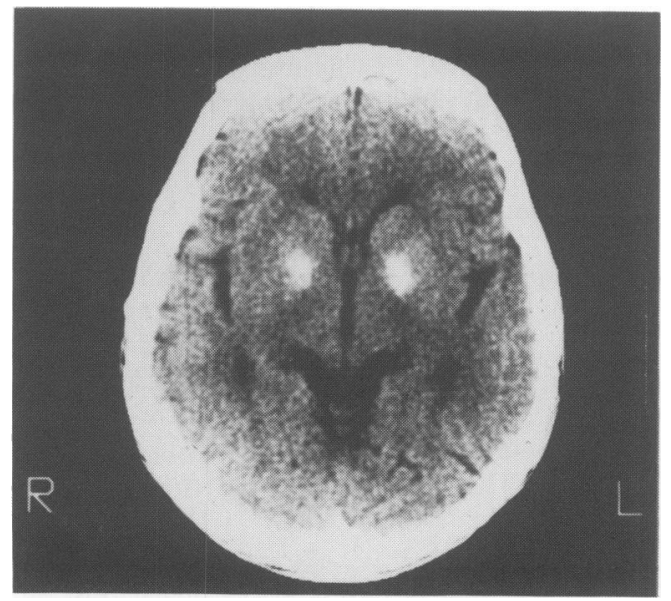

Figure 1 CT scan of brain showing basal ganglia calcification. 
picture of brief psychotic episodes evolving into a picture of dementia. He fitted into the description of late-onset ICBG described by Cummings et al. ${ }^{1}$

$\mathrm{He}$ had exposure to antipsychotic drugs for nearly 2 years but these were already discontinued for 3 years when he was referred to us. As his psychotic illness and treatment with antipsychotic drugs preceded the abnormal movements, it remains possible that part of his movement disorder can be attributed to tardive dyskinesia. Furthermore, excessive susceptibility of ICBG patients to the extrapyramidal effects of antipsychotics has been noted by various authors ${ }^{1,2}$ as some of these patients had also received antipsychotic treatment prior to the onset of their movement disorder. However, the patient's parkinsonian features could not be accounted for by tardive dyskinesia and is likely to be related to ICBG.

We hope that this report will heighten clinicians' awareness of this rare entity and we recommend that ICBG should be considered as a differential diagnosis in pscyhiatric patients with a movement disorder.

\title{
References
}

1. Cummings, J.L., Gosenfield, L.F., Houlihan, J.P. \& McCaffrey, T. Neuropsychiatric disturbances associated with idiopathic calcification of the basal ganglia. Biol Psychiatry 1983, 18: 591-601.

2. Francis, A.F. Familial basal ganglia calcification and schizophreniform psychosis. Br J Psychiatry 1979, 135: 360-362.

3. Boller, F., Boller, M. \& Gilbert, J. Familial idiopathic cerebral calcification. J Neurol Neurosurg Psychiat 1977, 40: 280-285.

4. Kane, J.M., Woerner, M. \& Lieberman, J. Tardive dyskinesia: Prevalence, incidence and risk factors. J Clin Psychopharmacol 1988, 8: $528-556$.
5. Kalamboukis, Z. \& Molling, P. Symmetrical calcification of the brain in the predominance in the basal ganglia and cerebellum. J Neuropathol Exp Neurol 1962, 21: 364-371.

6. Trautner, R.J., Cummings, J.L., Read, S.L. \& Benson, F Idiopathic basal ganglia calcification and organic mood disorder. Am J Psychiatry 1988, 145: 350-353.

7. Wylie, K.R. \& Harris, S.J. Idiopathic basal ganglia calcification presenting as hypomania. Int J Geriatr Psychiatry 1990, 51: $401-402$.

8. König, P. Psychopathological alterations in cases of symmetrical basal ganglia sclerosis. Biol Psychiat 1989, 25: 459-468.

\section{Pulmonary alveolar microlithiasis presenting with chronic cough}

\author{
Ipek Türktas, Sezen Saribas and Ferhun Balkanci ${ }^{1}$
}

\author{
Dr Sami Ulus Children's Hospital, Ankara, and 'Department of Radiology, Hacettepe University \\ Faculty of Medicine, Ankara, Turkey
}

\begin{abstract}
Summary: A case of a 10 year old boy with pulmonary alveolar microlithiasis is presented. Although most children with this disease are asymptomatic, our patient had persistent cough for more than 3 years. It is likely that his chronic cough was a direct consequence of the disease.
\end{abstract}

\section{Introduction}

Pulmonary alveolar microlithiasis (PAM) is a rare disease of unknown aetiology characterized by the presence of multiple microscopic stones within the pulmonary alveoli. Most patients with PAM have no symptoms and many others have minor or questionable symptoms when their disease is first diagnosed. The pattern in our patient follows that

Correspondence: I. Türktas, M.D., Bülten Sok 42/5, 06700 Kavaklidere, Ankara, Turkey.

Accepted: 20 July 1992 described in many cases in the literature but chronic cough has been seldom reported. ${ }^{1,2}$

\section{Case report}

A 10 year old boy had been complaining of dry cough for 6 months and his chest $\mathrm{X}$-ray showed bilateral dense miliary mottling. He was diagnosed as having miliary tuberculosis by a local physician. The cough persisted following 6 months of antituberculosis therapy and the chest X-ray remained unchanged. He was referred to our hospital in 